\title{
Analysis of 3GPP LTE-Advanced Cell Spectral Efficiency
}

\author{
Daniel Bültmann, Torsten Andre \\ and Rainer Schoenen \\ ComNets Research Group \\ RWTH Aachen University \\ Aachen, Germany \\ Email: $\{$ dbn,tae,rs $\} @$ comnets.rwth-aachen.de
}

\begin{abstract}
Multihop and multipoint transmissions are two of the main features towards an increased spectral efficiency for the LTE-Advanced mobile radio system. Fixed wireless relays with in-band backhauling are considered as a multihop technique in LTE-Advanced. Relays improve cell capacity and cell edge user performance depending on the deployment. In this paper, at first, results for the peak spectral efficiency of LTE-Advanced are presented and secondly, an analytical model to calculate the cell spectral efficiency of relay enhanced cell deployments in the context of the IMT-Advanced evaluation is presented. The developed model is applied to the LTE-Advanced system comparing different relay deployments with different frequency reuse schemes.
\end{abstract}

\section{INTRODUCTION}

In March 2008 the International Telecommunication Union - Radiocommunication Sector (ITU-R) issued a circular letter inviting proponents to submit candidate systems for the terrestrial components of the International Mobile Telecommunication Advanced (IMT-A) radio interface(s). With its publication a certification process was started to ensure that candidate Radio Interface Technologys (RITs) fulfill certain key performance requirements. Some of these well exceed the performance of current third generation mobile networks.

To ensure a fair and transparent evaluation process, the ITU$\mathrm{R}$ soon after March 2008 released a performance requirement specification [1] and guidelines for evaluation of candidate systems [2]. Performance requirements must be evaluated either analytically, by inspection, or by means of simulation. Cell spectral efficiency and Voice over IP (VoIP) capacity for instance must be evaluated by means of system level simulation. All proponents are required to submit a selfevaluation report along with the technology proposal. External evaluation groups serve to provide independent results to increase the confidence that the performance requirements are met.

The 3rd Generation Partnership Project (3GPP) has submitted a self evaluation report on LTE-Advanced along with the technology proposal for IMT-A. Within this paper the authors study the Cell Spectral Efficiency (CSE) of this IMT-A proponent. One of the new technology components specified for Long Term Evolution-Advanced (LTE-A) is the usage of multi-hop communication using fixed Relay Nodes (RNs). Relay nodes are a practical solution to cover large cell areas with a limited number of base stations. One benefit of relay nodes is that no expensive wired backhaul is required for their deployment making the provisioning of radio access over the area more cost-efficient.

The authors present an analytical approach to calculate the cell spectral efficiency for Relay Enhanced Cells (RECs) according to the IMT-A evaluation methodology taking into account the probabilistic Line of Sight (LoS)/Non Line of Sight (NLoS) selection of large scale radio link characteristics. The model is based on the results available in [3] and [4] and extends the models to include the distance-dependent LoS/NLoS probabilities.

The rest of the paper is structured as follows. In section II the authors present Peak Spectral Efficiency (PSE) results for LTE-A that provide an upper bound for the cell spectral efficiency under best (ideal) radio conditions. The results are compared to the requirements of the ITU-R and the selfevaluation report of the 3GPP. In section III the model for calculation of the cell spectral efficiency is presented and section IV provides results for the Urban Macro (UMa) scenario with one, three and no relay nodes per sector.

\section{A. Relaying in LTE-Advanced}

LTE-A is planned to support RNs transmitting their own control channels and cell specific reference signals, which allows them to be used both for capacity enhancement or area extension, solely depending on the position of the Relay Node (RN). According to [5] the wireless backhaul link for RNs shall be inband, which means that Base Stations (BSs) and RNs use the same resources in time multiplex which may lead to self-interference at the RN in uplink and downlink direction if no countermeasures are applied. In uplink direction self-interference caused by User Terminals (UTs) connected to RNs can be avoided by not assigning any uplink resources to UTs during the uplink transmission over the backhaul. In downlink direction it is not sufficient to not assign resources to any UTs connected to a RN, since at least Long Term Evolution (LTE) R8 UTs still depend on the reception of control channels and reference signals. In case of missing signals from the BS (or in this case a RN), UTs assume a lost connection and restart the connection sequence. 
To overcome this problem it is suggested to use Multicast Broadcast Single Frequency Network (MBSFN) subframes already introduced in LTE release 8 to maintain backwards compatibility. MBSFN allows to group UTs for multipoint reception, while UTs not assigned to this group ignore the subframes used for MBSFN. These subframes are then used to reserve downlink transmission areas for the backhaul preventing the UTs to lose their connections.

The MBSFN subframe enables a RN to transmit the Physical Downlink Control CHannel (PDCCH) in the first two symbols to supply its associated UTs. Afterwards the RN switches its transmission direction to receive data from its donor BS. Finally it switches again to transmit data to its UTs.

Here only relay deployments for capacity enhancement with a varying number of RNs per cell are considered to improve the CSE. Besides the relay deployment different frequency reuse schemes for Radio Access Points (RAPs) are considered, which essentially influence the CSE in scenarios with a large number of RNs.

\section{B. Frequency Reuse Schemes}

Frequency reuse schemes allow neighbored RAPs to use distinct sets of resources which leads to larger frequency reuse distances, e.g. the distance between two RAP that use the same set of resources. The application of frequency reuse allows to decrease the interference from co-channels and therefore to increase the Signal to Interference plus Noise Ratio (SINR) and finally the throughput. LTE was designed to support frequency reuse one schemes on which this paper concentrates. In [6] different frequency reuse schemes were suggested and compared. The approach is to partition the available resources and apply different relative transmit powers to the partitions. This concept is applied to RECs. While BSs and RNs use the same resources in time multiplex and, therefore, do not interfere, RNs and BSs cause co-channel interference each among themselves. Currently two frequency reuse one schemes, namely uniform frequency reuse (see Figure 1(a)) and soft frequency reuse (see Figure 1(b)) with 70-20-10 partitions, i.e. three partitions with $70 \%, 20 \%$, and $10 \%$ of the maximal transmit power, are compared next to hard frequency reuse. In hard frequency reuse resource partitions are distinct leading to increased frequency reuse distances. In case of hard frequency reuse the ITU-R requires to normalize the cell spectral efficiency taking into account the frequency reuse distances.

\section{Peak Spectral Efficiency of LTE-Advanced}

One of the requirements the ITU-R specified for IMT-A systems is the PSE. While the ITU-R requests the PSE to be measured above Physical (PHY) layer, we computed the PSE above Medium Access Control (MAC) layer to determine the user PSE, which allows us to use the obtained PSE as an upper bound for the computation of the CSE within the next section. Further it allows to compare the PSE results to the self evaluation by the 3GPP [5]. We believe that the Time Division

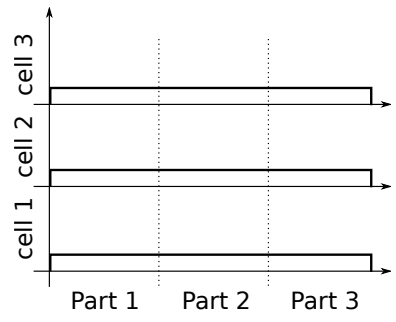

(a) Uniform frequency reuse.

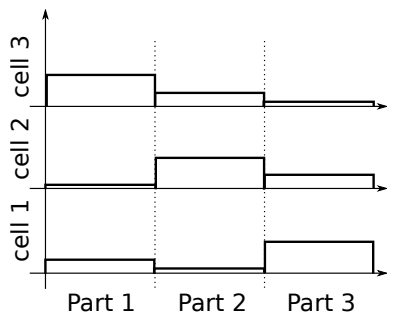

(b) Soft frequency reuse.
Fig. 1. Frequency reuse schemes.

Duplex (TDD) PSE figures of the 3GPP self-evaluation do not take the guard times into account properly. Uplink and downlink bandwidths are given by

$$
\begin{aligned}
& B_{\text {norm }}^{D L}=\frac{T_{D L}}{T_{U L}+T_{D L}} \cdot B \\
& B_{\text {norm }}^{U L}=\frac{T_{U L}}{T_{U L}+T_{D L}} \cdot B
\end{aligned}
$$

where $T_{U L}$ is the absolute uplink time in a radio frame and $T_{D L}$ the downlink time correspondingly. Both need to include the guard times of the system. 3GPP does not include the guard times in the bandwidth normalization, which leads to slightly higher results for TDD.

Nevertheless, comparable numbers result from different assumptions of flexible overheads, which we assume to be configurable in an acceptable way resulting in less overhead. In the uplink we assume a different configuration for the Physical Random Access CHannel (PRACH) as well as a reduced number of Resource Blocks (RBs) for the Physical Uplink Control CHannel (PUCCH), which increases the PSE.

TABLE I

ITU REQUIREMENTS AND RESULTS FOR LTE [BPS/HZ]

\begin{tabular}{lcccc}
\hline & \multicolumn{2}{c}{ Downlink } & \multicolumn{2}{c}{ Uplink } \\
Organization & FDD & TDD & FDD & TDD \\
\hline Requirement [1] & 15.0 & 15.0 & 6.75 & 6.75 \\
3GPP [5] & 16.3 & 16.0 & 8.4 & 8.1 \\
With TDD Guard Times & 16.3 & 15.8 & 8.5 & 8.1 \\
(SISO) & 4.5 & 4.3 & 4.4 & 4.0 \\
\hline
\end{tabular}

Table I shows the results both from the 3GPP self-evaluation and from the authors own calculations for $20 \mathrm{MHz}$ system bandwidth for LTE. Additionally, the PSE for SISO is included with respect to the computation of the CSE in Section IV. In case of FDD a total bandwidth of $40 \mathrm{MHz}$ is assumed. Further, Figure 2 shows the PSE depending on the allocated bandwidth for LTE-A. The assumptions that were made are as follows:

- 64QAM Modulation

- Code rate 1

- TDD frame configuration 1 and 3 for switching point periodicity of $5 \mathrm{~ms}$ and $10 \mathrm{~ms}$, respectively

- LTE: 4x4 MIMO (4 spatial streams) - LTE-A: 8x8

- Synchronization and reference signals according to [7] 
- Physical Broadcast CHannel (PBCH), PRACH, PDCCH, PUCCH according to [7]

LTE-A well exceeds the ITU requirements. It can be seen that for high bandwidth allocation the PSE is nearly constant, while for smaller bandwidths, constant overhead needed for the $\mathrm{PBCH}, \mathrm{PRACH}, \mathrm{PDCCH}$ and the PUCCH significantly reduce the PSE. Although we do not compute the PSE as requested by the ITU-R, it can be concluded that LTE-A fulfills the PSE requirement with our more restrictive computation.

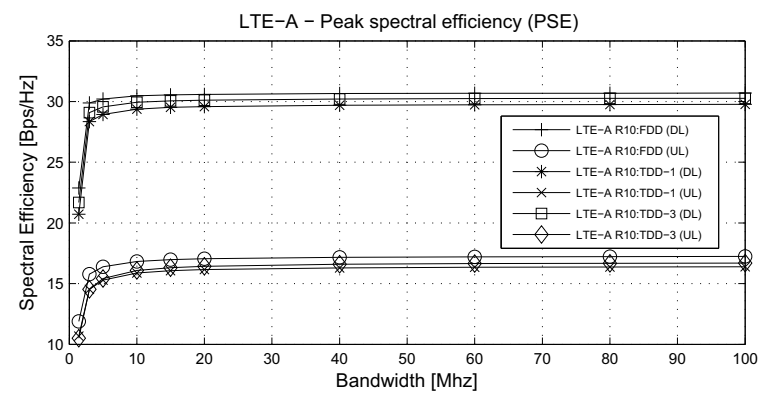

Fig. 2. Peak spectral efficiency for LTE-Advanced.

\section{Analytical MAC-LAyer Model for IMT-A SCENARIOS}

In this section an analytical model of a MAC-Layer suitable to calculate cell capacities for the downlink of multi-cellular scenarios, taking into account $\mathrm{LoS}$ and NLoS propagation condition probabilities, arbitrary BS placements, antenna patterns, and transmit powers, is developed. It requires an abstraction of the physical layer and relies on performance metrics of modulation coding schemes in terms of Frame Error Rates (FERs) acquired from link-level simulations. Shadow fading and fast fading effects as well as power control are currently neglected.

\section{A. SINR Computation} by

Generally, the received power at the UT position is given

$$
P_{R x}(x, y)=P_{T x} \cdot G_{U T}(\phi, \theta) \cdot G_{B S}(\phi, \theta) \cdot L_{P L}(d)
$$

where $P_{R x}$ is the receive power, $P_{T x}$ the total power emitted at the transmitter, $G_{U T}$ and $G_{B S}$ the possibly directional antenna gain at the UT and BS, respectively and $L_{P L}$ is the path loss depending on the distance $d$ between transmitter and receiver. In multicelullar scenarios the serving cell is selected according to the maximum $P_{R x}$ from a set of $M$ BSs. This can be expressed by

$$
s=\underset{j}{\arg \max }\left(P_{R x_{0}}, P_{R x_{1}}, \ldots, P_{R x_{M}}\right)
$$

Once the serving cell is determined, the downlink SINR is given by

$$
\operatorname{SINR}(x, y)=\frac{P_{R x_{s}}(x, y)}{\sum_{j \neq s} P_{R x_{j}}(x, y)+\eta}
$$

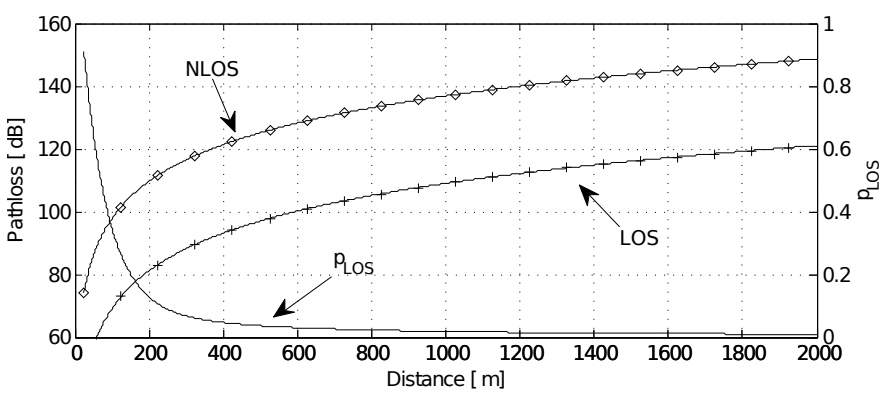

Fig. 3. Path loss and $p_{L o S}$ for UMa

where $\eta$ is the thermal noise level including the UT noise figure.

Radio link conditions between BS and UT can either be LoS or NLoS, which is determined by a LoS probability $p_{L o S}(d)$ depending on the distance $d$ as defined by the ITU-R [2]. Figure 3 illustrates the path loss and LoS probability for the UMa scenario.

The mean SINR at any given position also depends on the likelihood of LoS conditions to any of the $N$ BSs at this position. Let $p_{i}^{L o S}$ be the probability for link $i$ to have LoS, the permutation $j$ is denoted as

$$
\text { perm }_{j}=\left(p_{j, 1}, p_{j, 2}, \ldots, p_{j, M-1}, p_{j, M}\right), \quad j=1 \ldots 2^{M}
$$

where $p_{j, i}$ is the probability for link $i$ to have LoS. $M$ equals the number of RAPs. The probability $p_{p e r m, j}$ of perm $_{j}$ to occur is

$$
p_{\text {perm }, j}=\prod_{i=1}^{M} p_{i} \quad \forall j
$$

Hence the SINR at position $(x, y)$ can be computed by summing over the set of all permutations $\mathfrak{P}$ and weighting each computed SINR by its permutation's probability. The SINR at point $(x, y)$ is computed according to Equation 4 and the link assumptions associated with the permutation.

$$
\operatorname{SINR}(x, y)=\sum_{j \in \mathfrak{P}} p_{\text {perm }, j} \cdot \operatorname{SINR}_{j}(x, y)
$$

It shall be noted that the SINR calculation is non-linear and thus must be done separately for each point in the evaluated area.

\section{B. Capacity Calculation}

Given the SINR, the effective capacity is calculated according to Equation 8 based on the FER (assuming an Selective REJect (SREJ) Automatic Repeat Request (ARQ) [8]), where $C_{\text {total }}$ and $C_{\text {belowARQ }}$ is the capacity normalized to one symbol.

$$
C_{\text {total }}=C_{\text {below } A R Q} \cdot(1-F E R)
$$

The capacity calculation relies on two abstractions of the link-level of the investigated system:

1) The Adaptive Modulation and Coding (AMC) scheme must be taken into account in Equation 8. Based on the SINR an optimal Modulation and Coding Scheme 
(MCS) must be chosen, which can follow different optimization criteria, e.g. throughput maximization or delay minimization.

2) For each MCS a mapping between SINR and FER must exist, usually determined from link-level simulations.

When extending the model to relaying, it becomes necessary to associate an area element with either a BS or an UT depending on the optimization criterion. For throughput maximization in each permutation the capacity for all BSs as well as for all RNs according to Equation 9 is compared and the maximum chosen. The capacity for relays is computed by the reciprocal capacities of the single hops to take account of the additional resources that are needed on the first hop.

$$
\frac{1}{C_{\text {total }}}=\frac{1}{C_{\text {totalHop } 1}}+\frac{1}{C_{\text {totalHop } 2}}
$$

The cell capacity is computed assuming a proportional fair scheduling scheme which allows all UTs the same throughput:

$$
\frac{1}{C_{c e l l}^{\text {bit }}}=\frac{1}{A_{\text {cell }}} \sum_{x, y} \frac{1}{C_{\text {total }}(x, y)}
$$

\section{Complexity Reduction and Error Estimation}

Due to the large number of permutations ( $2^{M}$ for $M$ RAPs) and the resulting computational complexity the exact values can only be determined for small $M$.

To increase the feasible number of RAPs per scenario and thus reduce the computational complexity three steps are taken. Firstly, only the center site is evaluated instead of the whole scenario as required by the IMT-A evaluation guidelines. This is valid since only the downlink is investigated. Secondly, the total number of considered RAPs is reduced. Therefore, the following studies only include the first tier of interfering cells. This assumption has been validated with our system level simulator [9] and from Figure 4 it can be seen that the second tier of interferers only has limited impact on the SINR. Thirdly, the number of permutable RAPs is reduced, i.e. radio links from UTs to some RAPs are fixed to NLoS conditions, which leads to an upper limit for the CSE.

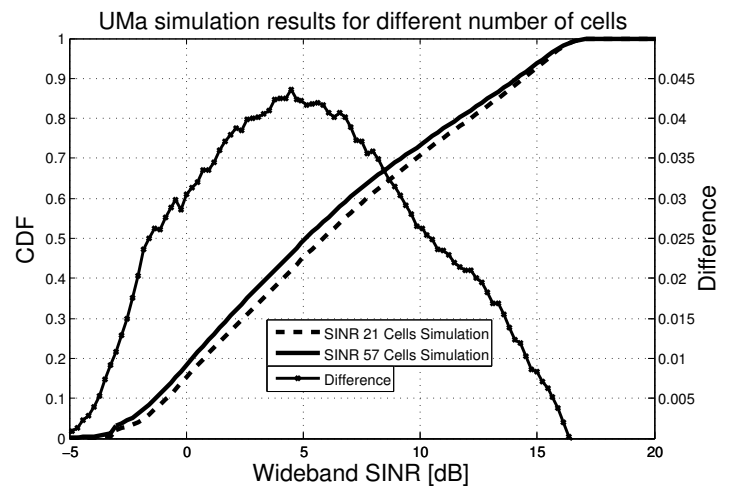

Fig. 4. Net capacity of LTE modulation and coding schemes.

The total error introduced by the third assumption depends on the absolute SINR error for a permutation where some link conditions are fixed and on the significance $\left(p_{\text {perm }, j}\right.$ in Equation 7) of these permutations. An upper limit for the total error with hexagonal RAP deployment will be derived in the following.

Let $N$ be the number of permuted RAPs, $M$ the total number of RAPs in the scenario. The reduced number of permutations is $2^{N}$ of $2^{M}$ permutations without complexity reduction. Therefore, the relative amount of the reduced number of permutations is

$$
\frac{2^{N}}{2^{M}}=2^{N-M} \rightarrow 0 \text { for } M \gg N
$$

It is assumed that for scenarios with a large number of RAPs the fraction of correctly computed permutations is neglectable. The approach is to derive a most representative permutation which can be used to compute the relative SINR which indicates a possible relative error after the throughput computation.

First permutation groups are introduced which group permutations of the same type. Two permutations are of the same type, if the number of included LoS links is the same. Within the permutation a one at the $i^{\text {th }}$ position indicates a LoS link between the UT and the $i^{\text {th }}$ BS, while a zero indicates a NLoS link. Let $X$ be a Random Variable (RV) that models the number of LoS links within a permutation. Then Equation 12 can be used to compute the relative cardinality of each permutation group where $x$ is the number of LoS links (the number of ones) and $M$ is length of the tuple, i.e. the number of RAP in the scenario.

$$
P(X=x)=\frac{\left(\begin{array}{c}
M \\
x
\end{array}\right)}{2^{M}}, \quad x=0 \ldots M
$$

Equation 12 assumes all permutations to be equally distributed. The only point for which this holds in a hexagonal scenario is at the cell center. There the LOS probabilities are independent of the distance because all interferers are equally distanced, i.e. the permutations within a permutation group have the same probability. Though Equation 6 leads to non-equally distributed probabilities for the permutations, the assumption of equally distributed permutation probabilities is useful to derive an upper bound of the error as will be motivated later on in this section.

Let the RV $Y$ model the number of LoS links within a permutation similar to $X$. While $X$ will only be associated with the equally distributed permutation groups' probabilities defined in Equation 12, $Y$ is associated with the permutation groups' probability based on the included link probabilities in Equation 13.

$$
P(Y=y)=p_{L o S}^{y} \cdot\left(1-p_{L o S}\right)^{(M-y)}, \quad y=0 \ldots M
$$

Equations 12 and 13 are now combined and multiplied with the number of permutations to derive the Probability Density Function (PDF) indicating the combined probability for each permutation group. Let $Z$ be a RV like $X$ and $Y$, but $\mathrm{Z}$ will be used to indicate the aggregated PDF. 
TABLE II

SCENARIO PARAMETERS

$$
P(Z=z)=P(X=z) \cdot P(Y=z) \cdot 2^{M}, \quad z=0 \ldots M
$$

A representative number of LoS links per permutation, which can be used to compute the relative error, is obtained by computing the expected value of $Z$ :

$$
E(Z)=\sum_{z=1}^{M} z \cdot P(Z=z)
$$

As mentioned above, links whose state are not altered are always assumed to be NLoS. This means that the computation of an exact SINR

$$
S I N R_{e}=\frac{R x_{F e e d}}{\sum_{i=0}^{L} R x_{L o S, i}+\sum_{i=0}^{N} R x_{N L o S, i}}
$$

is altered to an approximated SINR of

$$
S I N R_{a}=\frac{R x_{F e e d}}{\sum_{i=0}^{M} R x_{N L o S, i}}
$$

Since it is assumed that the UT is located at the cell center, the received powers are all the same, i.e. independent of the index $i$. This allows to easily determine the relative error $\epsilon$ made when computing the SINR.

$$
\epsilon=\left|\frac{S I N R_{a}-S I N R_{e}}{S I N R_{e}}\right|=\left|\frac{E(Z) \cdot\left(R x_{L o S}-R x_{N L o S}\right)}{(M-1) \cdot R x_{N L o S}}\right|
$$

The derivation of the relative error is only valid for the cell center as assumed above. Though this error can be used as an upper bound for the overall error. As stated above the error is assumed to be proportional to the number of LoS links within a permutation. Therefore, the point with the highest probability for an permutation including a maximum number of LoS experiences the highest error, which is at the cell center. At all other places this probability decreases, concluding that permutations including less LoS links are more probable, though these permutations result in an reduced error due to the number of reduced LoS links.

It is shown above that the error is nearly independent of the number of permutations. Nevertheless non-permutable RAPs are chosen to minimize their influence on the error. For RAPs which are far off the evaluated area have a small LoS probability which decreases the probability $p_{p e r m, j}$ and hence reduces the impact of the permutation compared to a RAP which is close to the evaluated area with a higher LoS probability. In case of 21 RAPs only permutations with less or equal than five LoS links contribute close to $100 \%$ to the mean SINR and are therefore significant. Further investigations revealed that only permutation groups with up to five LOS links are significant, i.e. only

$$
\frac{\sum_{i=0}^{5}\left(\begin{array}{c}
21 \\
i
\end{array}\right)}{2^{21}}=1.3 \%
$$

of the total permutations are significant. This deduction allows to reduce the computation complexity while further reducing the expected error. Though these conclusions are only valid

\begin{tabular}{ll}
\hline Parameter & \multicolumn{2}{c}{ Value } \\
\hline \multicolumn{2}{c}{ Link Level } \\
\hline Transmission scheme & SIMO 1x2 \\
HARQ & 1 transmission \\
Receiver type & MRC \\
Control channel overhead & 3 OFDM symbols \\
Channel model & AWGN \\
Allocated RBs & 50 \\
\hline & System Level \\
& UMa see [2] \\
\hline Pathloss & $2 G H z$ \\
Carrier Frequency & $49 d B m$ \\
BS Tx Power (20M Hz) & $30 d B m$ \\
RN Tx Power & $17 d B i$ \\
BS Borsesight Antenna Gain & see [2] \\
BS Antenna Pattern & $12^{\circ}$ \\
BS Antenna Downtilt & $0 d B i$ \\
RN Borsesight Antenna Gain & $0 d B i$ \\
UT Borsesight Antenna Gain & $0 d B i m$ \\
BS-BS Distance & $500 m$ \\
BS-RN Distance & $216.5 m$ \\
Avg. Car Penetration Loss & $9 d B$ \\
Feeder Loss & $2 d B$ \\
UT Noise Figure & $7 d B$ \\
Thermal noise & $-174 d B m / H z$ \\
AMC Scheme & Maximum Throughput \\
Scheduling & Proportional Fair \\
\hline &
\end{tabular}

for the scenario center, the expected number of relevant permutations is not assumed to alter significantly as long the evaluated area is not too distanced from the scenario center (or any place where the above assumptions hold, for the matter of fact).

\section{Cell Spectral EfFiciency of LTE-Advanced}

In this section the previously described model is applied to LTE-A and a comparison for the CSE of non-relaying, one relay and three relays deployments for the urban macro-cell scenario is presented.

\section{A. Scenario}

Table II lists the parameters for the evaluated scenario. The link level parameters were assumed to derive the SINR to FER mappings as shown in Figure 6 and assume the users to operate in high throughput mode, i.e. each user is assigned a large number of resource blocks for the individual transmission.

Figure 5 illustrates the scenario. The scenario consists of one tier of six BSs around the center cell with the according number of RNs.

Due to the computational complexity the second tier of BSs required by the ITU-R is omitted. Further the number of combinations is reduced to keep the computation feasible. The Inter Site Distance (ISD) is $500 \mathrm{~m}$, the RNs are placed at three fourth of the cell radius to increase the throughput at the cell edge, which is most efficient to increase the CSE. For the wireless backhaul of the RN 256QAM with code rate 1/1 is assumed neglecting eventually necessary retransmissions for now. 


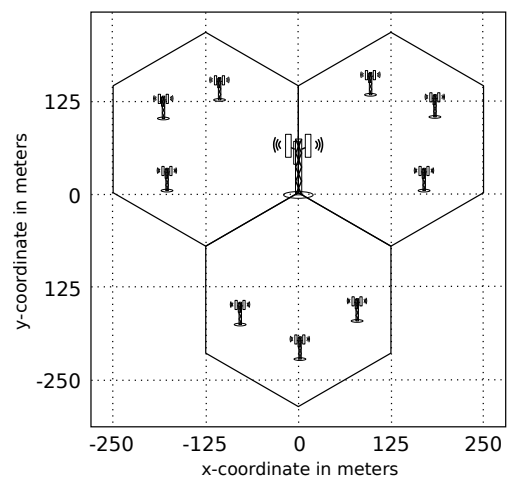

Fig. 5. RN deployment for UMa-1 (only the middle RN per cell) and UMa-3 (three RNs per cell)

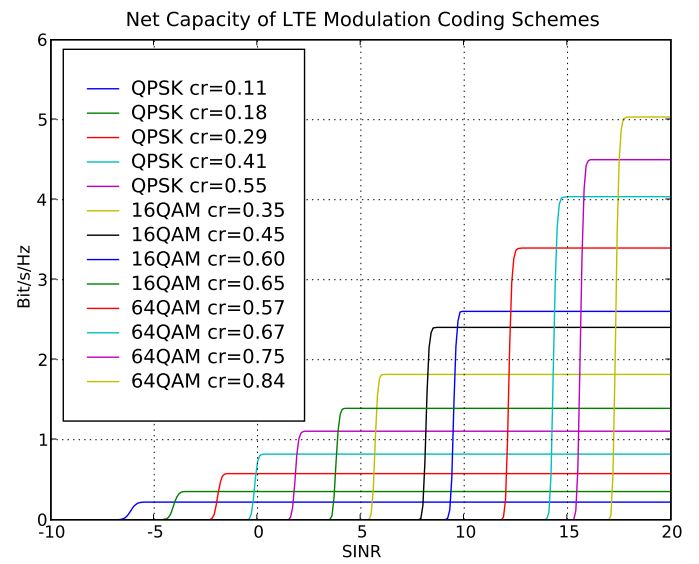

Fig. 6. Net capacity of LTE modulation and coding schemes.

\section{B. Results}

In this section, a comparison for the CSE of non-relaying, one relay and three relays deployments for the urban macrocell scenario is presented.

Table III lists cell spectral efficiencies for the different deployment options. The naming convention for the scenario short names is composed of four parts:

1) The three letter acronym for the scenario.

2) The number of relays per cell.

3) The deployed reuse scheme for the BSs where U, S, and $\mathrm{H}$ stand for Uniform Frequency Reuse (UFR), Soft Frequency Reuse (SFR), and Hard Frequency Reuse (HFR), respectively.

4) The deployed reuse scheme for the RNs with the according letter

The first two lines within the table show the error-free computation of the CSE and the computation based on the previously introduced assumptions. As can be seen the effective error is $1.7 \%$ which is less than half the estimated upper bound for the relative error.

Futher it can be seen that SFR between the BSs significantly increases the CSE, regardless of the number of deployed RNs. In all scenarios the CSE is further increased up to $14 \%$ when
TABLE III

NET CSE RESUlTS FOR $20 \mathrm{MHz}$ BANDWIDTH.

\begin{tabular}{lcccc}
\hline Scenario & FDD & $\begin{array}{c}\text { TDD } \\
{[\mathrm{bps} / \mathrm{I} z / \text { cell] }}\end{array}$ & $\begin{array}{c}\text { max. rel. SINR } \\
\text { error [\%] }\end{array}$ \\
\hline UMa-0-U & 1.15 & 1.13 & 2.2 & 0.00 \\
UMa-0-U & 1.17 & 1.14 & 2.2 & 3.82 \\
UMa-0-S & 1.82 & 1.76 & 2.2 & 3.82 \\
\hline UMa-1-U.U & 1.47 & 1.42 & 2.2 & 3.73 \\
UMa-1-S.U & 1.92 & 1.86 & 2.2 & 3.73 \\
\hline UMa-3-U.U & 1.41 & 1.37 & 2.2 & 3.68 \\
UMa-3-U.H & 1.76 & 1.70 & 2.2 & 3.68 \\
UMa-3-S.H & 2.09 & 2.03 & 2.2 & 3.73 \\
\hline
\end{tabular}

deploying $3 \mathrm{RNs}$ and up to $5 \%$ when deploying $1 \mathrm{RN}$. An important result is that with more than $1 \mathrm{RN}$ it is crucial to assign distinct resources to the RNs, otherwise there is no additional gain. Also it can be seen that the requirement of $2.2 \mathrm{bps} / \mathrm{Hz} / \mathrm{cell}$ cannot be reached. It must be noted here that only SISO links are taken into account within this analysis. Thus, it seems plausible that the requirements are easily reached if MIMO links are considered that boost performance in areas of high SINR.

\section{CONClusion}

This paper presents an overview of the relaying concept in LTE-A and proposes an analytical approach to derive the Cell Spectral Efficiency (CSE) for scenarios used in the IMTA evluation. The model is applied to Relay Enhanced Cell (REC) deployments with none, one and three RNs per cell in the Urban Macro (UMa) scenario. Furthermore, uniform and soft frequency reuse schemes were applied and the impact on the CSE was studied. It was shown that proper resource partitioning between RNs is important and that RNs increase the CSE by $5 \%$ to $14 \%$ with the selected deployment. The next step is to find optimal deployment scenarios for relays that yield maximum gain of the CSE.

\section{REFERENCES}

[1] ITU, "M.2134 : Requirements related to technical performance for IMTAdvanced radio interfaces," tech. rep., ITU, 2008.

[2] ITU, "M.2135 : Guidelines for evaluation of radio interface technologies for imt-advanced," Report M.2135, ITU-R, 2008.

[3] R. Schoenen and B. Walke, "On PHY and MAC performance of 3G-LTE in a multi-hop cellular environment," in Proceedings of the 3rd IEEE International Conference on Wireless Communications, Networking and Mobile Computing (WiCOM), (Shanghai, China), p. 6, Sep 2007.

[4] J. Habetha and J. Wiegert, "Network capacity optimisation, part 1: Cellular radio networks," vol. 0, (Aachen), pp. 125-132, Sep 2001.

[5] 3GPP TR 36.912, Feasibility study for Further Advancements for E-UTRA (LTE-Advanced), June 2010. v9.3.0.

[6] M. Bohge, J. Gross, and A. Wolisz, "Optimal power masking in soft frequency reuse based ofdma networks," in Proc. of European Wireless Conference (EW'09), (Alborg, Denmark), May 2009.

[7] 3GPP TS 36.311, Evolved Universal Terrestrial Radio Access (E-UTRA) Physical Channels and Modulation (Release 8), March 2009. v8.6.0.

[8] B. H. Walke, Mobile Radio Networks 2nd Edition. John Wiley \& Sons, 2002.

[9] D. Bültmann, M. Muehleisen, K. Klagges, and M. Schinnenburg, "openWNS - open Wireless Network Simulator," in 15th European Wireless Conference 2009 Electronic Proceedings, (Aalborg, Denmark), pp. 205210, VDE VERLAG GMBH, May 2009. 